\title{
Pontuações de Rastreamento em Hospitais: Uma Proposta de Avaliação Baseada em Históricos de Contexto
}

\author{
Rogério Albandes ${ }^{1}$, Roger Machado ${ }^{2}$, Adenauer Yamin ${ }^{1}$ \\ ${ }^{1}$ Programa de Pós-Graduação em Eng. Eletrônica e Computação (PPGEEC) \\ Universidade Católica de Pelotas (UCPel) - Pelotas - RS - Brasil \\ ${ }^{2}$ Instituto Federal de Educação, Ciência e Tecnologia Sul-rio-grandense (IFSul) \\ Câmpus Gravataí - Gravataí-RS-Brasil \\ \{rogerio.albandes, adenauer.yamin\}esou.ucpel.edu.br \\ rogermachadoeifsul.edu.br
}

\begin{abstract}
In doctors, mobility is part of their daily lives, so these professionals can spend periods without contact with the teams that support them in the treatment of patients. Long periods between communications can cause delays in performing procedures, prescribing drugs, etc. Based on this scenario, this work aims to design a proposal that integrates: (i) a platform for the acquisition of vital signs; (ii) an environment for contextual processing, which, through customizable rules, inference the patients' situation; and (iii) a textual and graphical visualization interface for these signals, which can be accessed through the IoT. As a source of vital signs, the MIMIC-III database, widely accepted by the international community for this purpose, is being used. In turn, a preliminary evaluation of the proposal with health professionals, using interviews, obtained positive feedback, pointing to the continuity of the research.
\end{abstract}

Resumo. Nos médicos a mobilidade faz parte de seu cotidiano, assim, é possivel que esses profissionais fiquem períodos de tempo sem contato com as equipes que lhes dão suporte no tratamento dos pacientes. Longos lapsos de tempo entre comunicações podem provocar retardos na realização de procedimentos, na prescrição de drogas, etc. Com base nesse cenário, este trabalho tem por objetivo a concepção de uma proposta que integra: (i) uma plataforma para aquisição de sinais vitais; (ii) um ambiente para processamento contextual, que através de regras personalizáveis faça a inferência da situação dos pacientes; e (iii) uma interface de visualização textual e gráfica desses sinais, que possa ser acessada pela IoT. Como fonte de sinais vitais, está sendo utilizada a base de dados MIMIC-III, amplamente aceita pela comunidade internacional para esse fim. Por sua vez, uma avaliação preliminar da proposta junto a profissionais de saúde, empregando entrevistas, obteve retorno positivo, apontando para continuidade da pesquisa.

\section{Introdução}

Considerando que a mobilidade constitui prática habitual no dia-a-dia dos médicos, implicando no trânsito por diferentes ambientes (hospitais, consultório, etc.), é possível que os mesmos fiquem consideráveis períodos de tempo sem contato com as equipes que lhe dão suporte no tratamento dos pacientes [Costa Dias 2015]. 
Por sua vez, estudos têm indicado que a frequência na comunicação entre os médicos e os outros profissionais de saúde nos hospitais constitui aspecto importante no tratamento de pacientes internados. Períodos mais longos entre comunicações podem provocar retardos na realização de procedimentos, na prescrição de drogas, etc., o que contribui para um possível aumento no período de internação [Rufino et al. 2012].

A premissa perseguida neste trabalho é explorar recursos da Internet das Coisas (IoT), tanto para aquisição de informações sobre os pacientes, como para realizar uma interoperação com a comunidade médica sempre que necessário. Esta interoperação será coordenada por procedimentos automatizados, regidos por mecanismos para Ciência de Contexto.

A Ciência de Contexto refere-se a um modelo no qual o sistema computacional é capaz de verificar os aspectos nos quais tem interesse e, quando necessário, reagir as suas alterações disparando procedimentos pertinentes. Esta abordagem materializa premissas da IoT, nas quais existe uma comunicação autônoma entre objetos inteligentes, utilizados pelos profissionais de saúde, cooperando para o avanços das suas diferentes atividades [Perera et al. 2014].

De acordo com [Sezer et al. 2018], para a construção de sistemas cientes de contexto em ambientes distribuídos, como é o caso da presente proposta, alguns desafios devem ser tratados: (i) aquisição do contexto a partir de fontes heterogêneas e distribuídas; (ii) processamento dos dados contextuais adquiridos; e (iii) as respectivas ações direcionadas aos dispositivos e pessoas envolvidas.

Considerando este cenário, este trabalho tem por objetivo a concepção de uma proposta que integra: (i) uma plataforma para aquisição de sinais vitais; (ii) um ambiente para processamento contextual, que através de regras personalizáveis faça a inferência da situação dos pacientes a partir de um histórico de contexto construído a partir de sinais vitais, e quando necessário envie notificações para os profissionais de saúde envolvidos; e (iii) uma interface de visualização textual e gráfica destes sinais, que possa ser acessada de forma remota.

Para tanto, na concepção da proposta será explorada a arquitetura de software do middleware EXEHDA, particularmente do seu subsistema dedicado a processamento contextual, o qual será empregado na inferência da situação dos pacientes.

A expectativa com a proposta é permitir que os médicos possam remotamente antecipar diagnósticos e a consequente prescrição de procedimentos. Além disto, entendese que a pesquisa em andamento tem potencial para contribuir com a redução do tempo de internação.

Este artigo está organizado em oito seções. A segunda e terceira Seção apresentam conceitos julgados interessantes quando da revisão de literatura em relação à proposta. Na quarta Seção são discutidos os trabalhos relacionados. Na quinta Seção é introduzido o middleware EXEHDA destacando suas principais funcionalidades envolvidas para a obtenção da Ciência de Contexto. A sexta Seção apresenta a proposta abordando suas principais características. Na sétima Seção é discutida a prototipação e os testes realizados com a proposta. Por fim, a oitava e última Seção apresenta as considerações finais e os trabalhos futuros. 


\section{Trabalhos Relacionados}

Durante o esforço de pesquisa foram identificados diversas abordagens relacionadas ao monitoramento remoto de pacientes, dentre as quais foram selecionados cinco trabalhos. Para sua seleção o trabalho deveria contemplar os seguintes aspectos: (i) monitorar sinais vitais; (ii) oferecer suporte para operações remotas tanto de sensoriamento como de atuação (envio de alertas, etc.); e (iii) considerar o emprego de Ciência de Contexto.

O trabalho de [Jaiswal et al. 2018] apresenta um modelo que automatiza a coleta, entrega e processamento de dados vitais dos pacientes com a ajuda de um dispositivo de borda e do container Docker. Segundo o autor, o monitoramento de saúde e os aplicativos de resposta à emergências baseados em IoT exigem uma latência e um atraso menor quando da troca de informações. As informações são trocadas entre o servidor de borda, a nuvem e o dispositivo do usuário, o que afeta diretamente o desempenho. Para alcançar o objetivo proposto no trabalho, é utilizado o Raspberry pi como dispositivo de borda, para otimizar o processo de análise dos dados dos sensores, com isto é possível trabalhar com baixa largura de banda, baixa latência e com redes congestionadas.

No artigo de [Dridi et al. 2017], é proposta a plataforma SM-IoT, uma plataforma baseada em IoT para cuidados de saúde inteligentes e personalizados, dedicada aos pacientes, bem como aos cuidadores. O objetivo desta plataforma é melhorar o monitoramento remoto do paciente e promover os serviços de saúde. A plataforma SM-IoT é capaz de coletar dados de fontes de informação heterogêneas, integrá-los usando uma web semântica flexível, armazená-los na nuvem para análise posterior, visualizar esses dados com interfaces amigáveis e facilitar seu compartilhamento levando em conta seu aspecto de privacidade.

No trabalho de [Karthikeyan et al. 2015], é proposto um sistema de automação hospitalar distribuído, autônomo, flexível e de baixo custo. Constituído de servidores e sensores que podem ser facilmente configurados. Uma placa Intel Galileo, com uma placa Wi-fi integrada, funciona como um servidor web, que permite o acesso de pessoas autorizadas na mesma rede LAN, utilizando um computador pessoal ou, remotamente, através de Wi-Fi ou 3G/4G utilizando um smartphone. O Sistema beneficia não apenas os pacientes que recebem um tratamento mais eficiente mas também aos médicos que podem agilizar seus esforços para atender a um número maior de pacientes. A ideia principal deste sistema é um monitoramento contínuo dos pacientes e um controle de instrumentos, via internet.

A proposta de [Ahmed 2017] apresenta um framework genérico de Health-IoT que contém um Sistema de Apoio à Decisão Clínica (Clinical Decision Support SystemsCDSS), para fornecer um sistema de monitoramento de saúde auto-adequado e personalizado para idosos em ambiente doméstico. O framework é focado principalmente nos sensores de suporte, nos meios de comunicação, na comunicação segura e confiável de dados, no armazenamento baseado em nuvem e nos acessos remotos dos dados. O CDSS é utilizado para fornecer um relatório personalizado sobre o estado de saúde das pessoas com base na observação diária dos sinais vitais. Um conjunto de regras pré-determinadas é usado para classificar parâmetros de saúde individuais e o Raciocínio Baseado em Casos (Case-Based Reasoning - CBR) é aplicado para gerar o estado geral de saúde de um usuário. 
Em [Maia et al. 2015] é apresentada a EcoHealth (Ecossistema de Dispositivos de Health Care), uma plataforma de middleware que integra sensores corporais heterogêneos para permitir o monitoramento remoto de pacientes e a melhoria de diagnósticos médicos. O seu objetivo principal é integrar informações obtidas a partir de tais sensores heterogêneos para fins de monitoramento, processamento, visualização e armazenamento desses dados, bem como de notificação e atuação referentes a condições atuais de pacientes e seus sinais vitais. O projeto da EcoHealth é baseado em várias tecnologias Web bem estabelecidas (HTTP, REST e EEML) com o intuito de padronizar e simplificar o desenvolvimento de aplicações no contexto de IoT, minimizando assim problemas de compatibilidade e de interoperabilidade entre fabricantes, protocolos proprietários e formatos de dados

Dentre os principais diferencias da atual proposta temos o emprego do Early Warning Score (EWS), que é um reconhecido padrão internacional para Pontuações de Rastreamento e Acompanhamento de sinais vitais, o qual tem sido utilizado em abordagens não automatizadas, não sendo contemplado nos trabalhos relacionados.

Outro diferencial diz respeito ao uso de um middleware, o qual somente é empregado pelo trabalho relacionado [Maia et al. 2015]. Para o tratamento do desafio de prover suporte a Ciência de Contexto às aplicações na IoT, vem se destacando o emprego de middlewares, os quais são inseridos entre as infraestruturas computacionais e as aplicações [Pires et al. 2014]. Os middlewares, por meio de interfaces de alto nível, permitem a interoperabilidade de diferentes dispositivos da IoT, disponibilizando dentre outras funcionalidades, um meio padronizado para o acesso aos recursos disponíveis nos mesmos.

\section{Sistemas para Pontuações de Rastreamento e Acompanhamento}

Os sinais vitais são sinais médicos que indicam o status das funções vitais (sustentadoras da vida) do corpo humano. Essas medidas são tomadas para ajudar a avaliar a saúde física geral de uma pessoa, fornecer pistas para possíveis doenças e mostrar progresso em direção à recuperação. Dentre os sinais vitais os considerados principais são: Temperatura, Pulso, Pressão Arterial, Frequência respiratória, Oximetria de Pulso e Escala AVPU

Existem sistemas chamados de Pontuações de Rastreamento e Acompanhamento (track-and-trigger scores), estes sistemas calculam uma pontuação que reflete o estado de saúde de um paciente em relação a seus sinais vitais. Vários sistemas de pontuação são utilizados internacionalmente [Bleyer et al. 2011].

Dentre esses sistemas, um dos mais utilizados é o EWS, o qual é baseado nos sinais vitais frequência respiratória, saturação de oxigênio, temperatura, pressão arterial, pulso/frequência cardíaca e resposta AVPU [Commission et al. 1999]. Faixas de valores foram estabelecidas em cada sinal vital (vide tabela 1) e adotado um valor para cada uma. Assim, o cálculo do EWS consiste na soma dos scores de cada Sinal Vital.

\section{Medidas de Similaridade em Históricos de Contexto}

Os pacientes, a medida que têm diferentes procedimentos terapêuticos em andamento, seus sinais vitais oscilam ao longo do tempo, constituindo uma série histórica. 
Tabela 1. EWS Computation

\begin{tabular}{l|c|c|c|c|c|c|c}
\hline EWS Sinal Vital Score & $\mathbf{3}$ & $\mathbf{2}$ & $\mathbf{1}$ & $\mathbf{0}$ & $\mathbf{1}$ & $\mathbf{2}$ & $\mathbf{3}$ \\
\hline SpO2 & $<85$ & $85-95$ & $90-92$ & $>92$ & & & \\
\hline Temperatura & & $>38,5$ & $38-38,9$ & $36-37,9$ & $35-35,9$ & $34-34,9$ & $<34$ \\
\hline PA Sistólica & & $<34$ & & $100-199$ & $80-99$ & $70-79$ & $<70$ \\
\hline Freq. Cardíaca & $>129$ & $110-129$ & $100-109$ & $50-99$ & $40-49$ & $30-39$ & $<30$ \\
\hline Freq. Respiratória & $>35$ & $31-35$ & $21-30$ & $9-20$ & & & \\
\hline AVPU & & & & Alerta & Verbal & Dor & S/resposta \\
\hline
\end{tabular}

O perfil de estabilidade, crescimento ou decréscimo dos sinais vitais de um paciente em particular, pode ser comparado com padrões já estabelecidos por médicos, estabelecendo assim, por níveis de similaridade, uma expectativa de tendência por um quadro clínico.

O nível de proximidade com relação a um determinado padrão, na presente proposta, será computado pela aplicação recorrente de uma métrica para medida de distância, considerando a evolução dos diferentes sinais vitais [Cha 2007].

Um dos mais antigos conceitos de medida de distância é a Distância Euclidiana (Euclidean Distance). Em matemática, Distância Euclidiana, ou distância métrica, é a distância entre dois pontos, que pode ser provada pela aplicação repetida do teorema de Pitágoras.

\section{Middleware EXEHDA}

O EXEHDA consiste de um middleware baseado em serviços, o qual visa criar e gerenciar um ambiente computacional largamente distribuído, bem como promover a execução de aplicações cientes de contexto sobre ele. O middleware vem sendo explorado em frentes de pesquisa que tratam desafios da IoT [Souza et al. 2018].

O EXEHDA possui uma organização composta por um conjunto de células de execução, conforme pode ser observado na Figura 1. Cada célula, no que diz respeito ao provimento de Ciência de Contexto, contempla um Servidor de Contexto (SC), diversos Servidores de Borda (SB) e/ou Gateways.

Os Gateways coletam informações contextuais, provenientes de sensores físicos ou lógicos e tem a finalidade de tratar a heterogeneidade dos diversos tipos de sensores, em aspectos tanto de hardware como de protocolo, transferirem estas informações coletadas de forma normalizada aos Servidores de Borda. No EXEHDA os Gateways são implementados sobre um hardware embarcado específico para a finalidade de interoperar com sensores e atuadores.

No EXEHDA o processamento das informações contextuais é distribuído, ficando uma parte com o Servidor de Borda, e outra com o Servidor de Contexto (vide Figura 1).

Os dados recebidos pelos diversos Servidores de Borda são transmitidos ao Servidor de Contexto que os gerencia e realiza as etapas de armazenamento e processamento contextual. O Servidor de Contexto pode combinar os dados provenientes dos Servidores de Borda com informações históricas, que ficam registradas no Repositório de Informações Contextuais. Uma discussão mais ampla das diferentes funcionalidades tanto do Gateway, quanto dos Servidores de Borda está disponível em [Souza et al. 2018], 


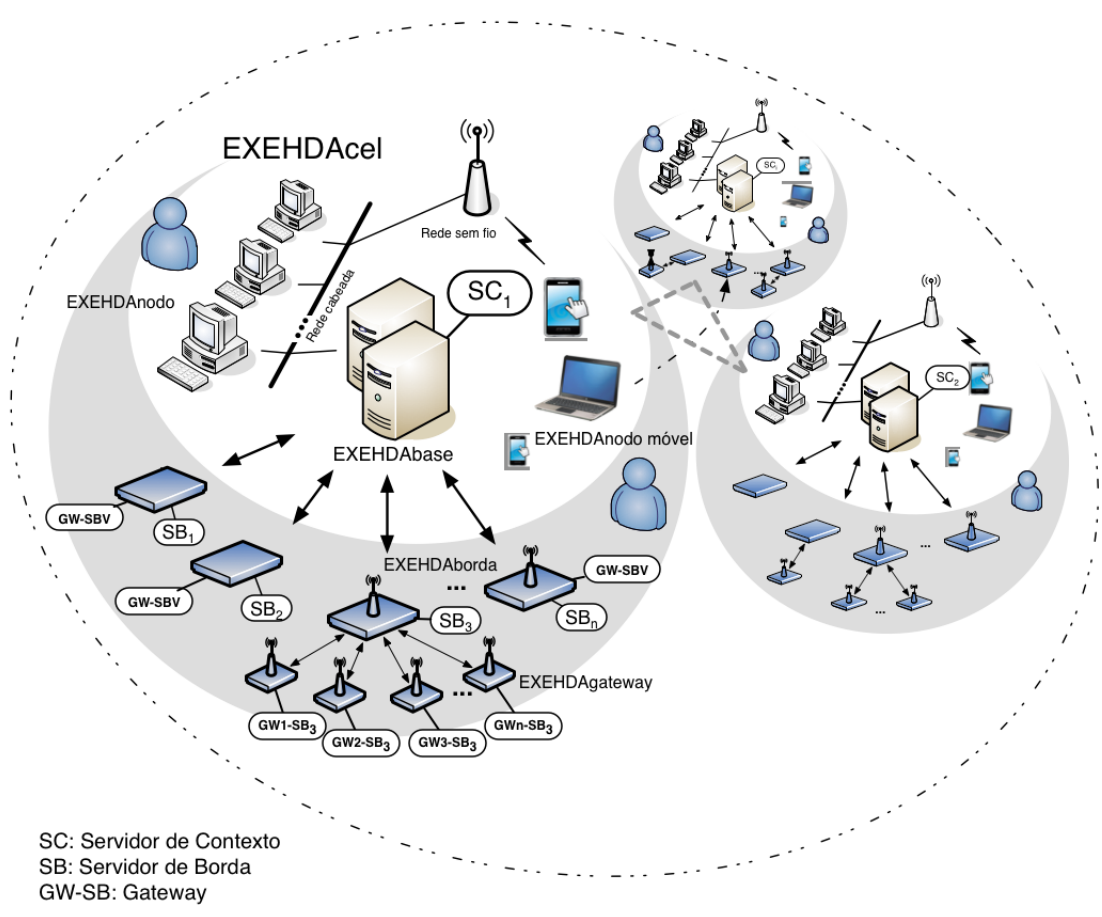

Figura 1. Ambiente para IoT gerenciado pelo EXEHDA

por sua vez, uma avaliação das diferentes potencialidades do Servidor de Contexto pode ser encontrada em [Lopes et al. 2014].

\section{Arquitetura e Funcionalidades da Proposta}

A arquitetura de de software concebida para a proposta $I^{2}$ VSM está apresentada na Figura 2. Na continuidade desta seção são tratadas as funcionalidades dos diferentes módulos, sendo discutidos seus perfis operacionais.

\subsection{Bloco de Interoperação com o Ambiente}

O Bloco de Interoperação do Ambiente é constituído pelo Módulo API_MultiMonitor, Módulo de Dispositivos e Módulo de Comunicação. Esse bloco opera sobre um Gateway Nativo do middleware EXEHDA.

O Módulo API_MultiMonitor contempla a entrada dos sinais vitais à partir de monitores paramétricos comerciais, possui uma RESTful API que permite a qualquer fabricante tornar disponíveis seus dados a proposta. O Módulo de Dispositivos é responsável por receber informações de sensores de sinais vitais, por meio de um system-ona-chip(SOC), direcionado a IoT, composto por uma ESP32, possuindo um programa em Python que coleta os dados vindos dos sensores. Por sua vez, o Módulo de Comunicação é responsável por transferir/receber informações e comandos do Bloco de Processamento de Borda.

\subsection{Bloco de Processamento de Borda}

Dois módulos formam o Bloco de Processamento de Borda, que é instanciado sobre o Servidor de Borda do EXEHDA. Módulo de Comunicação, o qual é responsável por in- 


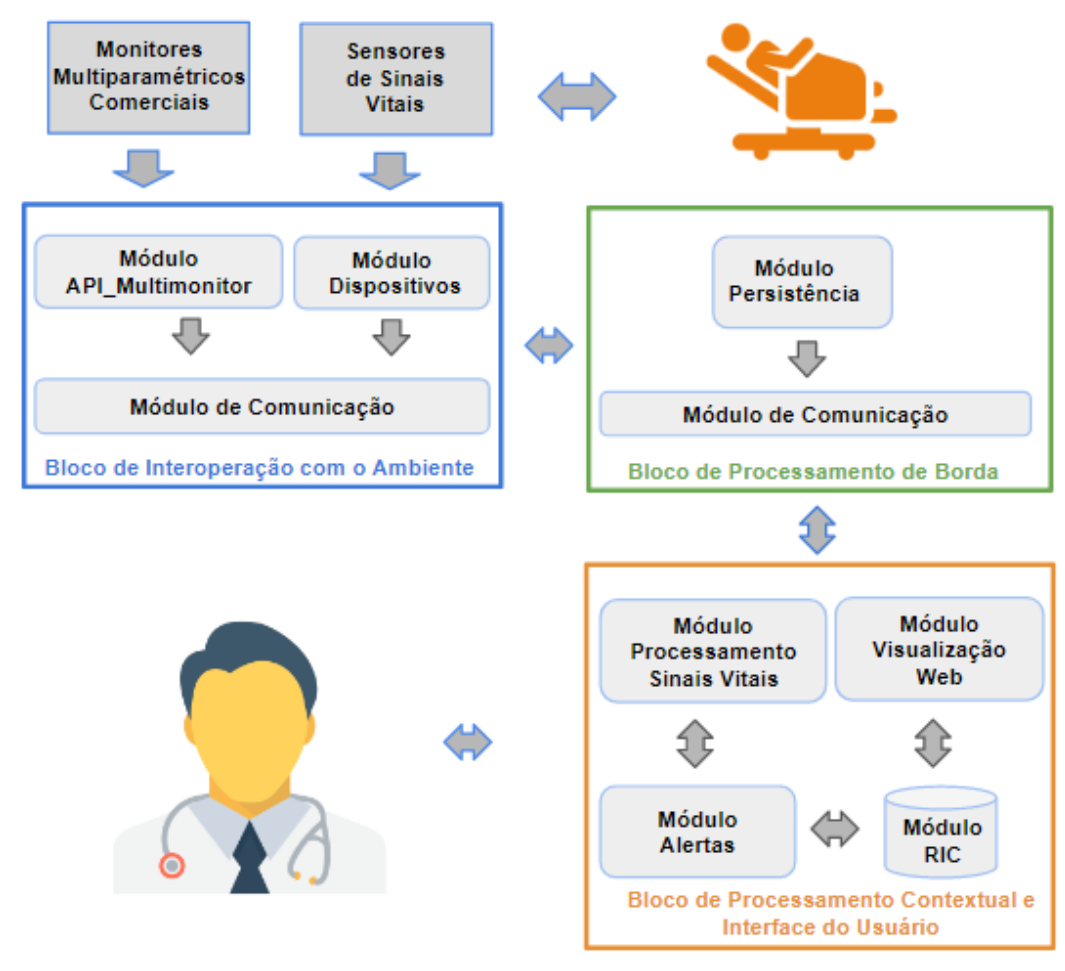

Figura 2. Arquitetura de software

teroperar com o Bloco de Processamento Contextual e Interface do Usuário, esta funcionalidade é instanciada no Módulo de Interoperação do Servidor de Borda do EXEHDA. Já o Módulo de Persistência, tem por objetivo realizar uma persistência temporária caso a conexão Internet com o Bloco de Processamento Contextual e Interface do Usuário seja perdida. Esta funcionalidade é instanciada sobre o Módulo Persistência Local do Servidor de Borda do EXEHDA.

\subsection{Bloco de Processamento Contextual e Interface do Usuário}

O Bloco de Processamento Contextual e Interface do Usuário é constituído pelo Módulo de Processamento dos Sinais Vitais, Modulo de Visualização Web, Módulo de Alertas e o Repositório de Informações Contextuais, operando sobre um Servidor de Contexto do middleware EXEHDA.

\section{Módulo de Processamento dos Sinais Vitais}

O processamento de dados contextuais se dá no Módulo de Processamento dos Sinais Vitais, onde os dados são recebidos e uniformizados. Após padronizados estes dados passarão pelo conjunto de regras que irão definir a situação do paciente. Neste módulo são tratadas todas as regras referentes ao disparo de alertas, baseado nos sinais vitais coletados. Integram o conjunto de regras: (i) regras baseadas em padrões internacionais, no caso o EWS; (ii) regras definidas pelo médico usuário, que irão traduzir especificidades baseadas em sua experiência profissional e/ou particularidades do paciente; e (iii) a distância entre padrões pré-definidos e o comportamento dos sinais vitais do paciente em questão.

O escore EWS é utilizado por padrão pela proposta para a geração dos alertas. Os gatilhos e alertas são diferentes para cada hospital e, a proposta permite que além do 
escore EWS, também o médico defina seus próprios gatilhos e correspondentes alertas. Será empregado como base os gatilhos utilizados pelo Norfolk and Norwich University Hospital ou NNUH. Segundo o NNUH os médicos devem ser acionados para uma revisão quando o score EWS for igual ou superior a 5, caso maior ou igual a 6 os médicos necessitam atender o paciente imediatamente (dentro de $30 \mathrm{~min}$ ).

O médico, usuário da proposta pode configurar seus templates de regras utilizando suas definições, as regras de padrões estabelecidos ou ainda um conjunto híbrido de regras - o que torna flexível a configuração de seus alertas personalizados. A configuração de regras personalizadas (vide Figura 3) possui uma interface intuitiva, chamada de Cadastro de Templates, onde o médico define quais sinais vitais irá utilizar, seus valores, operadores relacionais (igual, diferente, maior, menor, maior ou igual, menor ou igual) e os operadores lógicos (and e or) para concatenar os diferentes sinais vitais. Com isto o médico cria um template para cada situação de sua especialidade. Este template recebe um nome e ficará armazenado para uso posterior em qualquer paciente.

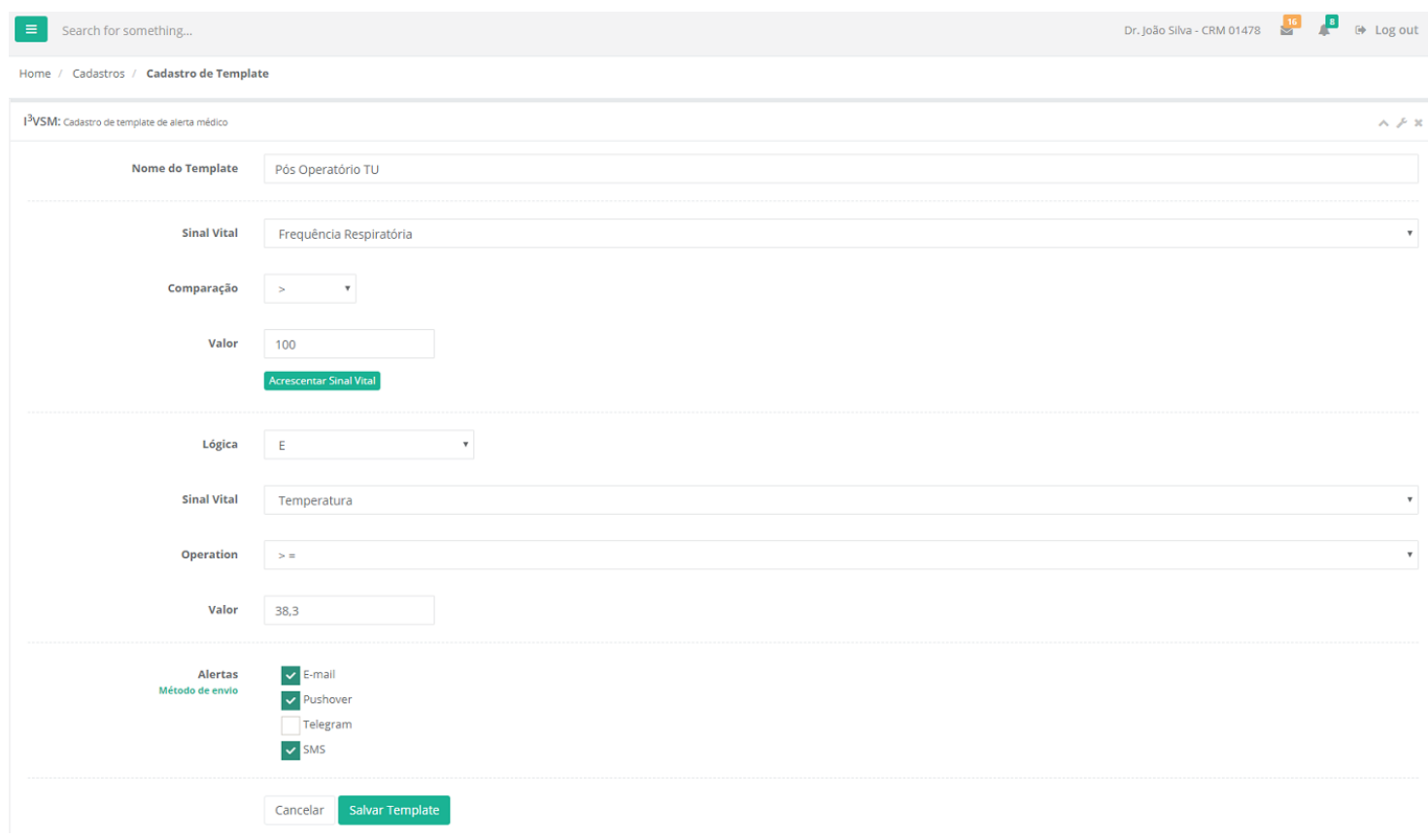

Figura 3. Tela de Cadastro dos Templates

Como terceira opção, além de regras para avaliar limites associados aos sinais vitais, é oferecido ao médico a possibilidade de especificação de padrões de comportamento com relação aos sinais vitais, os quais serão comparados com aqueles coletados dos pacientes. Esse padrão, após discussões envolvendo os profissionais de saúde, contemplou três possibilidades, definidas a cada procedimento de medição de sinais vitais: (i) Estável: o valor medido não flutuou, significativamente, em relação a medição anterior; (ii) Crescente: o valor medido do sinal vital é maior que o último valor registrado e (iii) Decrescente: o valor do sinal vital medido é menor que o último registrado.

O médico, tendo como critério o perfil do paciente, estabelece um número de amostras dos sinais vitais, obtidas em sequência, constituindo assim um histórico de dados contextuais do paciente. Este histórico de contextos será considerado para comparação com os padrões previamente estabelecidos. Essa comparação será realizada empregando 
as métricas definidas na Seção 4. A proposta irá facultar ao médico, salvar, com ou sem edição, um conjunto de sinais vitais de um paciente e associá-lo a um padrão que será empregado em futuras comparações.

Para tratar a tendência da situação do paciente, no decorrer do tempo foi estabelecida uma norma que servirá para medir a distância da variação do sinal vital no tempo $\Delta$ overtime, com a variação estabelecida pelo médico quando da definição do padrão $\Delta_{\text {standard }}$.

A interface da proposta, permite que o médico realize o cadastro de padrões da variação dos sinais vitais (aumentando, estável ou diminuindo), com o objetivo de ser alertado quando a situação do paciente estiver próxima a este padrão. Considerando que o valor mínimo, quando as séries são iguais, é zero e o máximo, quando as séries tem tendências totalmente opostas, é 4,24, foi adotado, para o envio de alertas de similaridade, sempre que o valor for inferior a 2 .

\section{Módulo de Alertas}

Nesta proposta, o papel dos alertas é preponderante, considerando as características da atividade dos profissionais da saúde quanto à mobilidade e períodos prolongados, sem a possibilidade de atender a ligações telefônicas. Alertas serão emitidos à equipe responsável pelo paciente seguindo regras por eles estabelecidas e/ou indicadores, internacionalmente aceitos. A proposta explora 2 serviços de mensagens que utilizam a Internet como meio: Pushover e Telegram; e um outro, denominado SMSGateway, que utiliza o serviço de Short Message Service (SMS) da rede GSM (Global System for Mobile Communications) de telefonia celular.

\section{Módulo Visualização Web}

O Módulo Visualização Web é responsável por toda interface visual da proposta. Suas funções vão das rotinas de login até as visualizações de dashboards de pacientes e telas que mostram dados importantes.

O Módulo Visualização Web foi dividido em componentes menores, conforme figura 4. São eles: Autenticação, Logs, Banco de Dados, Front End Web, Conector Nuvem e Administração e Configuração.

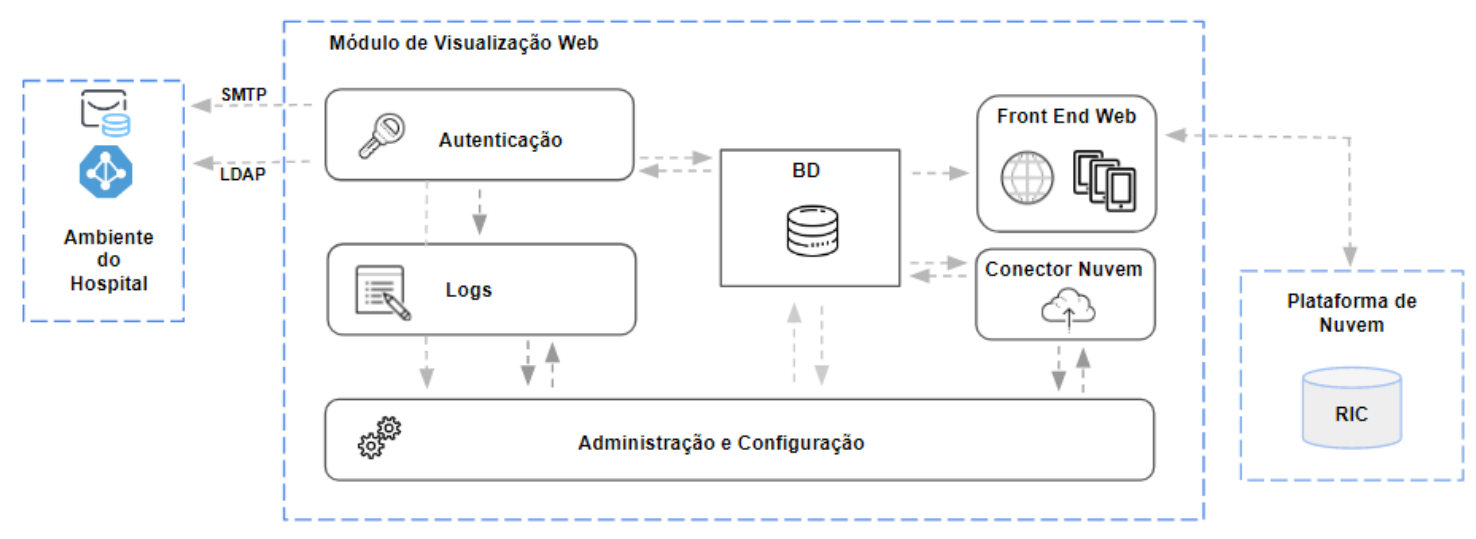

Figura 4. Arquitetura do módulo de Visualização Web

Considerando a otimização de espaço neste artigo as definições sobre os componestes do Módulo Visualização Web, podem ser obtidos em [Albandes 2019]. 


\section{Avaliação da Proposta}

Nos hospitais brasileiros é comum a medida dos sinais vitais quando da troca de turno das equipes de enfermagem, que se dá de $6 \mathrm{~h}$ em $6 \mathrm{~h}$. No banco de dados MIMIC-III as coletas são realizadas de $15 \mathrm{~min}$ em $15 \mathrm{~min}$. Objetivando adaptar o banco de dados à realidade da proposta foram excluídos os dados que estão fora destes valores: 00h00min, 06h00min, $12 \mathrm{~h} 00 \mathrm{~min}$ e $18 \mathrm{~h} 00 \mathrm{~min}$.

Com o objetivo de avaliar o funcionamento do Módulo de Processamento dos Sinais Vitais, foi utilizado o MIMIC-III já adaptado à realidade brasileira e ao escopo deste trabalho, sendo configurados os seguintes parâmetros: (i) Indice EWS for superior a 5 (chamado de alerta amarelo); (ii) Indice EWS for igual ou superior a 6 (chamado de alerta vermelho); e (iii) Alerta configurado pelo médico, aumento na frequência cardíaca, associada a febre. Foram processados 1.000 pacientes sendo gerados 250 alertas amarelo, 193 alertas vermelho e 12 alertas, considerando os parâmetros configurados pelo médico.

Por sua vez, para avaliação junto aos profissionais de saúde foi utilizado o $T e$ chnology Acceptance Model (TAM), um modelo, proposto por [Davis et al. 1989], que possui a vantagem de ser específico para tecnologia da informação e tem uma forte base teórica, além do amplo apoio empírico. O modelo sugere que, quando os usuários são apresentados a uma nova tecnologia, vários fatores influenciam sua decisão sobre como e quando eles a usarão, notadamente: (i) Utilidade percebida (Perceived Usefulness - PU) é o grau em que uma pessoa acredita que usar um determinado sistema aumentaria seu desempenho no trabalho; e (ii) Facilidade de uso percebida (Perceived Ease of Use - PEOU) - é o grau em que uma pessoa acredita que usar um determinado sistema estaria livre de esforço. Desta forma, foi elaborado um questionário cujas questões estão mostradas na tabela 2, empregando a escala Likert: Discordo totalmente; Discordo parcialmente; Indiferente; Concordo parcialmente e Concordo totalmente.

Tabela 2. Questionário TAM respondido pelos médicos

\begin{tabular}{c|l}
\hline Construto & \multicolumn{1}{c}{ Afirmativa } \\
\hline \multirow{2}{*}{ Facilidade de } & 1 - Considero a utilização da $I^{2}$ VSM clara e objetiva. \\
\cline { 2 - 2 } uso percebida & 2 - O preenchimento do Cadastro de Templates da $\mathrm{I}^{2}$ VSM é fácil e intuitivo. \\
\cline { 2 - 2 } & 3 - Considero as tecnologias de alertas de fácil utilização. \\
\hline \multirow{2}{*}{$\begin{array}{c}\text { Utilidade } \\
\text { Percebida }\end{array}$} & 4 - A utilização da $\mathrm{I}^{2}$ VSM melhoraria a ronda médica. \\
\cline { 2 - 2 } & 5 - A utilização da $\mathrm{I}^{2}$ VSM permitiria um melhor atendimento dos pacientes. \\
\cline { 2 - 2 } & 6 - A utilização da $\mathrm{I}^{2}$ VSM teria potencial para reduzir tempo de internação. \\
\hline
\end{tabular}

O questionário de avaliação desenvolvido foi aplicado a 10 médicos de 3 hospitais da cidade de Pelotas-RS, sendo apresentado aos mesmos uma demonstração da proposta, sendo os alertas, gerados à partir do MIMIC-III, usando o EWS e também os parâmetros configurados por eles, o que os aproximou do real funcionamento da proposta.

É fundamental avaliar se o questionário utilizado na pesquisa consegue inferir ou medir aquilo a que realmente se propõe, justamente, para dar relevância à pesquisa. O Coeficiente Alfa de Cronbach é uma medida bastante utilizada de confiabilidade (avaliação da consistência interna dos questionários) para um conjunto de indicadores de construto. O Coeficiente Alfa de Cronbach mede a correlação entre as respostas em um questionário, 
considerando a análise do perfil das respostas dadas. É calculado a partir do somatório da variância dos itens individuais e da soma da variância de cada avaliador [Taber 2018].

$\mathrm{Na}$ literatura encontra-se que os valores aceitáveis de alfa, variam de 0,70 a 0,95. Um baixo valor de alfa pode ser devido a um baixo número de perguntas, baixa interrelação entre itens ou construções heterogêneas. Se alfa for muito alto, pode sugerir que alguns itens sejam redundantes, pois estão testando a mesma pergunta, mas com uma aparência diferente. É recomendado um valor alfa máximo de 0,90 [Streiner 2003]. Considerando os dados obtidos no questionário, se obteve o valor do Alfa de Cronbach igual a 0,876 .

Baseado no valor obtido para o Alfa de Cronbach, superior a 0,7 , podemos considerar que a avaliação feita pelos profissionais de saúde, seguindo a metodologia da proposta TAM, possui uma considerável confiabilidade, podendo ser entendida como um instrumento representativo da opinião médica.

\section{Considerações finais}

O comportamento da arquitetura da proposta quando do emprego das diferentes funcionalidades, providas pelos Blocos da Arquitetura, se mostrou promissor em aspectos de estabilidade, tempo de resposta e produção de relatórios.

A expectativa é permitir que os profissionais de saúde possam antecipar diagnósticos e procedimentos médicos, o que dentre outros aspectos, tem potencial para reduzir o tempo de internação.

Os resultados obtidos junto à médicos no que diz respeito a avaliação da Utilidade e Facilidade de Uso percebidas (TAM), mostram-se promissores e apontam para a continuidade das pesquisas da proposta, enquanto uma abordagem que emprega o middleware EXEHDA na área da saúde.

Dentre os trabalhos futuros previstos tem-se o desenvolvimento de um aplicativo nativo para smartphones e a construção de uma API para integração com os sistemas legados dos hospitais.

\section{Referências}

Ahmed, M. U. (2017). An intelligent healthcare service to monitor vital signs in daily life-a case study on health-iot. Int. J. Eng. Res. Appl.(IJERA), 7(3):43-55.

Albandes, R. (2019). Node mcu [online]. Último acesso 23 Abril 2020.

Bleyer, A. J., Vidya, S., Russell, G. B., Jones, C. M., Sujata, L., Daeihagh, P., and Hire, D. (2011). Longitudinal analysis of one million vital signs in patients in an academic medical center. Resuscitation, 82(11):1387-1392.

Cha, S.-H. (2007). Comprehensive survey on distance/similarity measures between probability density functions. City, 1(2):1.

Commission, A. et al. (1999). Critical to success: the place of efficient and effective critical care services within the acute hospital. Audit Commission London.

Costa Dias, E. (2015). Condições de trabalho e saúde dos médicos: uma questão negligenciada e um desafio para a associação nacional de medicina do trabalho. Rev. bras. med. trab, 13(2). 
Davis, F. D., Bagozzi, R. P., and Warshaw, P. R. (1989). User acceptance of computer technology: a comparison of two theoretical models. Management science, 35(8):9821003.

Dridi, A., Sassi, S., and Faiz, S. (2017). A smart iot platform for personalized healthcare monitoring using semantic technologies. In Tools with Artificial Intelligence (ICTAI), 2017 IEEE 29th International Conference on, pages 1198-1203. IEEE.

Jaiswal, K., Sobhanayak, S., Turuk, A. K., Bibhudatta, S. L., Mohanta, B. K., and Jena, D. (2018). An iot-cloud based smart healthcare monitoring system using container based virtual environment in edge device. In 2018 International Conference on Emerging Trends and Innovations In Engineering And Technological Research (ICETIETR), pages 1-7. IEEE.

Karthikeyan, S., Devi, K. V., and Valarmathi, K. (2015). Internet of things: Hospice appliances monitoring and control system. In Green Engineering and Technologies (IC-GET), 2015 Online International Conference on, pages 1-6. IEEE.

Lopes, J. L., Geyer, C. F. R., Barbosa, J. L., Pernas, A. M., and Yamin, A. C. (2014). A middleware architecture for dynamic adaptation in ubiquitous computing. Journal of Universal Computer Science, 20(9):1327-1351.

Maia, P., Baffa, A., Cavalcante, E., Delicato, F. C., Batista, T., and Pires, P. F. (2015). Uma plataforma de middleware para integraçao de dispositivos e desenvolvimento de aplicaç oes em e-health.

Perera, C., Zaslavsky, A., Christen, P., and Georgakopoulos, D. (2014). Context aware computing for the internet of things: A survey. IEEE Communications Surveys and Tutorials, 16(1):414-454.

Pires, P. F., Cavalcante, E., Barros, T., Delicato, F. C., Batista, T., and Costa, B. (2014). A platform for integrating physical devices in the internet of things. Proceedings of the 12th IEEE International Conference on Embedded and Ubiquitous Computing, pages 234-241.

Rufino, G. P., Gurgel, M. G., Pontes, T. d. C., and Freire, E. (2012). Avaliação de fatores determinantes do tempo de internação em clínica médica. Revista Brasileira Clínica Médica, 10(4):291-297.

Sezer, O. B., Dogdu, E., and Ozbayoglu, A. M. (2018). Context-aware computing, learning, and big data in internet of things: a survey. IEEE Internet of Things Journal, $5(1): 1-27$.

Souza, R., Lopes, J., Geyer, C., Cardozo, A., Yamin, A., and Barbosa, J. (2018). An architecture for iot management targeted to context awareness of ubiquitous applications. Journal of Universal Computer Science, 24(10):1452-1471.

Streiner, D. L. (2003). Starting at the beginning: an introduction to coefficient alpha and internal consistency. Journal of personality assessment, 80(1):99-103.

Taber, K. S. (2018). The use of cronbach's alpha when developing and reporting research instruments in science education. Research in Science Education, 48(6):1273-1296. 